\title{
IMPROVING THE METHOD OF SOLAR RADIATION DURABILITY DETERMINATION OF CABLE PRODUCTS
}

\author{
Vladimir Rybakov ${ }^{1}$, and Elvira Sokolova ${ }^{1, *}$ \\ ${ }^{1}$ Tomsk Polytechnic University, 634050, Tomsk, Russia
}

\begin{abstract}
The authors suggest an extra physic-mechanical procedure to determine durability of cable goods to solar radiation exposure. The test check was carried out using the standard and the proposed procedures. The results of two tests were compared and analyzed. The use of the proposed technique allows improving the validity and reliability of the laboratory experiment conducted in a real production environment and exclude the low-quality cable items. The only disadvantage of the proposed technique is its labor intensiveness.
\end{abstract}

\section{Introduction}

One of the key factors negatively affecting the exploitative characteristics is solar radiation. It leads to early aging and wear of a cable jacket and requires the replacement of cables, which is uneconomical and leads to some additional financial expenses. The existing technique intended to assess sunlight durability of cables is imperfect, due to the fact only visual observation is carried out during the test. Thus, defective goods may be allowed for their use in industry.

The goal of the research is to improve the existing traditional technique used for durability assessment of cable goods exposed to solar radiation.

Research objectives are as follows:

1) To carry out a standard test procedure to determine the deterioration process in cable items.

2) To carry out the proposed procedure with physic-mechanical test, which is considered to be crucial in this research due to its ability to improve accuracy and efficiency of durability assessment.

3) To compare the results of two tests aiming to confirm the accuracy of the proposed testing procedure.

\section{Materials and Methods}

\footnotetext{
*Corresponding author: weiberd@tpu.ru
} 
To improve the technique concerning the assessment of solar radiation impact various cables were chosen as research objects. Selection of certain brands is determined by the necessity of their quality verification.

The tested cables are used as power cables for outdoor applications and explosion/fire hazardous structures. Various polymeric materials are used to produce insulation and jackets for these cables, providing they have different mechanical and chemical properties. It is to be mentioned that the composition of polymeric mixtures are kept in secret by the producers.

One of the most important criteria relative to operation properties is cable durability to sunlight exposure. Therefore, an external cable jacket is made to provide the protection against external various factors, among which solar radiation is. Thus, the outer jacket has been chosen as the study object.

The resistance to sunlight is determined as the ability of a cable jacket made of polymers to keep the desired mechanical and chemical properties over a long period. Solar radiation is one of the factors having significant impact on degradation, which influences polymer properties negatively. The most destructive is high-energy radiation having small wavelength [3]. Ionizing radiation as well as ultraviolet and visible parts of light spectrum of wavelengths belongs to these negative effects. The vulnerability of polymers to sunlight is defined by the presence of chromophore chemical groups capable to absorb light.

\section{Standard procedure for deterioration of jackets assessment caused by solar radiation}

A standard method of deterioration assessment of electrical products is in a visual observation and testing signs of destruction after exposure to solar radiation in a climatic chamber. The exposure lasts from 5 to 10 days depending on the purpose. In a Heat, Moisture and Solar Radiation Chamber (HMSRC) specific conditions are kept.

The inference about durability or vulnerability to sunlight exposure is based on visual detecting of cracks, color change or intermediate deterioration reactions products emissions.

\section{Physical-mechanical test}

Physical-mechanical tests contribute to the increase of accuracy and effectiveness of the method defining cable durability to sunlight exposure since they define this characteristic both qualitatively and quantitatively. The main indices of durability to sunlight in this work are unit elongation and tensile strength. The comparison of these two values before and after being exposed to the sunlight allows to assess the deterioration and the aging rate.

As test samples shovel-shaped pieces (according to State Standard IEC 60811-1-12011) were chosen (fig. 1).

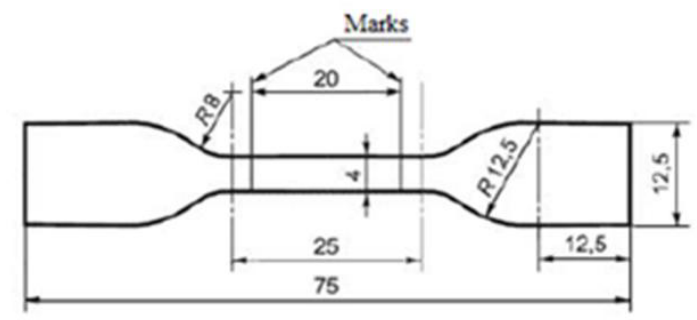

Fig. 1. Shovel-shaped sample. 


\section{Calculations}

Tensile strength and elongation values are calculated using the following expressions: $\sigma_{p}=\frac{P}{S}$, where $\mathrm{P}$ is the force applied to a sample, [kilogram-force]; $\mathrm{S}$ is the area of transverse section of the sample, $\left[\mathrm{mm}^{2}\right]$.

$$
\sigma_{\mathrm{p}}=\frac{15.8}{7.52}=2.101 \mathrm{kgs} / \mathrm{mm}^{2}
$$

$\theta=\frac{\left(1_{\mathrm{i}}-1_{0}\right)}{1} \cdot 100 \%$, where $l_{0}, l_{i}$ are the lengths of measuring section before the test and at rupture.

$$
\theta=\frac{63-20}{20} \cdot 100 \%=215 \%
$$

Arithmetic mean value is accepted as the calculation result.

\section{Result analysis}

The standard test according to State Standard 20.57.406-81 did not reveal any defects in the samples, like cracks, color change or depolymerization products emission. The external appearance of the tested sample has not changed. Thus, all types of cables have successfully passed the test.

Tension test results are shown in Fig.2. (bar chart). One can see, tensile strength values of aged samples are below the same results for the samples, which were not exposed to radiation. It can be assumed that there is an impact of solar radiation on mechanical strength of the jackets.

For the sample of HF(AF) jacket it is notable, tensile strength has dropped by $18 \%$. Elongation at rupture values of aged samples have risen by $34 \%$ after light exposure.
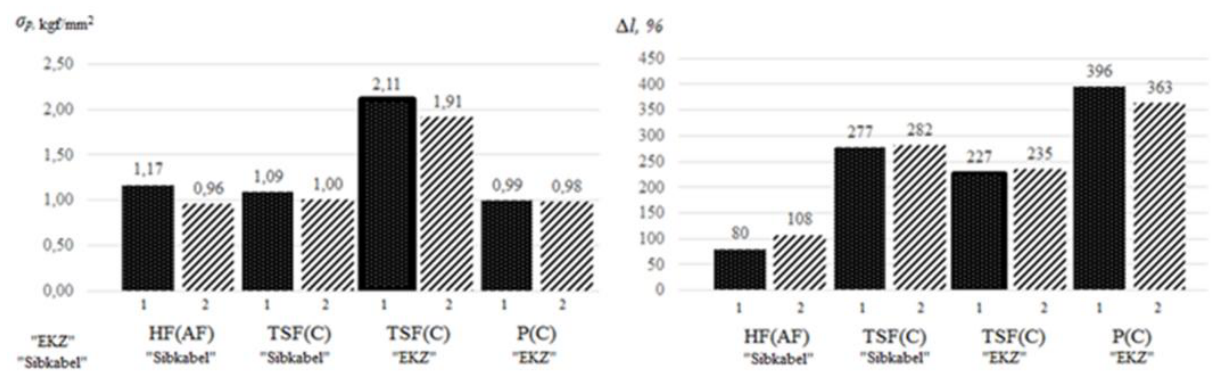

Fig. 2. Tensile strength changing and elongation at rupture changing of samples after solar radiation exposure: 1 - initial state values, 2 - values after aging in a climatic chamber.

For polymeric insulation noted that the increase in frequency, intensity increases abruptly discharges and reduces the mean time to breakdown, leading to insulation breakdown.

For the samples of TSF $(\mathrm{C})$ cable jackets made by two different manufacturers there is 8 $-9 \%$ drop of tensile strength after the light exposure.

Elongation values are $2-3 \%$ higher for aged samples. It should be noted that the tensile strength absolute values of initial samples made by "EKZ" are $95 \%$ higher, than the values of those made by "Sibkabel". Wherein, elongation values of initial "EKZ" samples are 18 $\%$ lower, than of those made by "Sibkabel".

The last jacket sample unlike the previous ones is made from thermosetting mixture, (rubber) and thus is subject of concern to our research. Experiment results state $1 \%$ change 
of tensile strength. Average elongation value of aged samples is $8 \%$ less than of the initial ones.

Upon the results of the test the cable jacket №1 Hold Cab EPR LV HF(AF) 4x2,5ок(N)1 is admitted to be insufficiently durable to solar radiation impact on physic-mechanical properties of a make. The degradation of tensile is about $18 \%$, which is the same value as the analogous one for thermal aging of this cable and is equal to $40 \%$ according to technical documents [6]. The change of elongation value for thermal aging is also $40 \%$.

Cable products like Hold Cab EPR LV TSF(C), made by different companies have shown the same durability to solar radiation, and the impact on physic-mechanical properties is comparable to thermal aging. Thermal deterioration causes $25 \%$ decline of mechanical properties [6].

A blend compound of rubber №4 Hold Cab EPR LV P(C) may contain vulcanizing agents and activators leftovers, which are able to maintain cross-linkage at $50^{\circ} \mathrm{C}$, which is the temperature being kept during the whole aging process. However, the impact of these components is lesser and is comparable to vulcanization irregularity within a sample

\section{Conclusion}

The obtained results concerning break durability and elongation at break allows stating the necessity of the standard technique improvement. The improved technique will increase the precision and efficiency of the resistance assessment of cables to solar radiation.

We can conclude that the proposed technique aimed at the research of the cable jackets resistance taking into account their physical-mechanical properties is perspective in terms of scientific and practical significance. In accordance with the standard test technique [5] all the produced items were recognized as resistant to solar radiation. The research with applying the proposed technique, however, showed the produced items being exposed to solar radiation have some defects like photochemical destruction and procure. Photochemical destruction of cable products leads to their precocious aging and the necessity to replace them and procure causes the flexibility decrease. Thus, the use of the proposed technique allows improving the validity and reliability of the laboratory experiment conducted in a real production environment and exclude the low-quality cable items. Thereby, the only disadvantage of the proposed technique is its labor intensiveness, since the majority of tests should be carried out manually.

\section{References}

1. A. Garganeev, A. Leonov, V. Merkulov, D. Charkov, IOP Conf. Ser.: Mater. Sci. Eng., A. A. Gerasimenko, 1 (1987)

2. A. A. Gerasimenko, Corrosion, aging and biological protection of machines, equipment and installations V. 2 (Mashinostroenie, Moscow, 1987)

3. L. S. Pinchuk, A.S. Neverov Polymer film containing corrosion inhibitors (Chemistry, Moscow, 1993)

4. State Standard IEC 60811-1-1-2011, The general testing methods of insulation materials and jackets of electrical and optical cables. (Publishing House of standards, Moscow, 2013)

5. State Standard 20.57.406-81, Electronic engineering product, quantum electronics products and electromechanical products. Test methods (Publishing House of standards, Moscow, 1982)

6. Specification 16.K73.130, (2015) 